\title{
Histone deacetylase inhibitors upregulate Rap1GAP and inhibit Rap activity in thyroid tumor cells
}

\author{
Xiaoyun Dong, Christopher Korch ${ }^{1}$ and Judy L Meinkoth
}

\author{
Department of Pharmacology, School of Medicine, University of Pennsylvania, Philadelphia, 19104 Pennsylvania, USA \\ ${ }^{1}$ UCCC DNA Sequencing and Analysis Core, University of Colorado Cancer Center, University of Colorado, Denver, \\ 80045 Colorado, USA \\ (Correspondence should be addressed to J L Meinkoth; Email: meinkoth@ upenn.edu)
}

\begin{abstract}
Increases in Rap activity have been associated with tumor progression. Although activating mutations in Rap have not been described, downregulation of Rap1GAP is frequent in human tumors including thyroid carcinomas. In this study, we explored whether endogenous Rap1GAP expression could be restored to thyroid tumor cells. The effects of deacetylase inhibitors and a demethylating agent, individually and in combination, were examined in four differentiated and six anaplastic thyroid carcinoma (ATC) cell lines. Treatment with the structurally distinct histone deacetylase (HDAC) inhibitors, sodium butyrate and trichostatin A, increased Rap1GAP expression in all the differentiated thyroid carcinoma cell lines and in four of the six ATC cell lines. The demethylating agent, 5-aza-deoxycytidine, restored Rap1GAP expression in one anaplastic cell line and enhanced the effects of HDAC inhibitors in a second anaplastic cell line. Western blotting indicated that Rap2 was highly expressed in human thyroid cancer cells. Importantly, treatment with HDAC inhibitors impaired Rap2 activity in both differentiated and anaplastic tumor cell lines. The mechanism through which Rap activity is repressed appears to entail effects on the expression of multiple Rap regulators, including RapGEFs and RapGAPs. These results suggest that HDAC inhibitors may provide a tractable approach to impair Rap activity in human tumor cells.
\end{abstract}

Endocrine-Related Cancer (2011) 18 301-310

\section{Introduction}

Thyroid cancer is the most prevalent endocrine cancer in the United States and worldwide (Tuttle et al. 2007). Malignant thyroid tumors derived from thyroid follicular cells include well-differentiated papillary and follicular thyroid carcinomas (PTC and FTC respectively), poorly differentiated thyroid carcinomas, and undifferentiated or anaplastic thyroid carcinomas (ATC). Together, PTC and FTC account for $>90 \%$ of all thyroid tumors, with PTCs comprising around $80 \%$ of all thyroid tumors. The prognosis for patients with differentiated thyroid tumors is good, with a 10 -year survival rate of 92-98\%. As well-differentiated thyroid tumors evolve to a less differentiated state, they become refractory to therapy because of widespread invasion and distant metastasis. ATC exhibits a disease-specific mortality of $97 \%$ with a mean survival of 6 months post-diagnosis. Novel therapies directed at
ATC would constitute a major advance in the treatment of thyroid cancer.

Mammalian Rap proteins (Rap1a/b and Rap2a/b/c) are members of the Ras superfamily of small GTPases. Cellular Rap activity is tightly controlled by guanine nucleotide exchange factors (RapGEFs) that activate Rap and GTPase activating proteins (RapGAPs) that inactivate Rap. The RapGAP family comprised several members including Rap1GAP/RapGAPII, SPA1/SIPA1, and E6TP1/SIPA1L1, of which Rap1GAP is highly expressed in thyroid cells (Tsygankova et al. 2004). Rap1GAP inhibits both Rap1 (Tsygankova et al. 2004, 2007, Vuchak et al. 2009) and Rap2 activity (McLeod et al. 2004, Tsygankova et al. 2010). Accumulating evidence indicate that RapGAPs function as tumor suppressors. Downregulation of E6TP1 by human papilloma virus protein E6 contributes to epithelial cell immortalization (Gao et al. 2001) 
and cervical cancer (Gao et al. 1999, 2002). SPA-1-deficient mice exhibit a spectrum of myelodysplastic disorders similar to chronic myelogenous leukemia (Ishida et al. 2003). The RaplGAP gene maps to $1 \mathrm{p} 35-36$, a chromosomal region subject to copy number alterations in many human tumors (Nagai et al. 1995, Williamson et al. 1997). Loss of heterozygosity for the RaplGAP gene has been reported in pancreatic and thyroid carcinomas (Zhang et al. 2006, Nellore et al. 2008).

Rap1GAP expression is downregulated in many human tumors including thyroid carcinomas (Tsygankova et al. 2007). The expression of Rap1GAP was further decreased in invasive versus benign lesions (Nellore et al. 2008, Zuo et al. 2010). These findings support a role for decreased expression of Rap1GAP in tumor promotion. Consistent with this notion, restoration of Rap1GAP expression in FTC cells impaired cell migration, invasion, and anchorage-independent proliferation (Tsygankova et al. 2007). In PTC cells, overexpression of Rap1GAP impaired cell migration, invasion, and proliferation (Zuo et al. 2010). In this study, we explored whether endogenous Rap1GAP expression could be restored to thyroid tumor cells. Rap1GAP expression was upregulated by treatment with histone deacetylase inhibitors in differentiated and ATC cell lines, while demethylases were less effective. Importantly, treatment with HDAC inhibitors was sufficient to decrease Rap activity. Increases in Rap activity have been shown to promote metastasis (Itoh et al. 2007, Bailey et al. 2009). Our findings suggest that HDAC inhibitors may constitute a therapeutic approach that could be used to decrease Rap activity, which might be beneficial in attenuating tumor spread.

\section{Materials and methods}

\section{Reagents}

Rap1GAP, Rap1GAPII, Spa-1 Rap1, Rap2, C3G, and actin antibodies were purchased from Santa Cruz (Santa Cruz, CA, USA). Anti-acetyl histone H3 was purchased from Millipore (Lake Placid, NY, USA). The DNA demethylating agent 5 -aza- 2 -deoxycytidine (5-Aza) and histone deacetylase inhibitor trichostatin A (TSA) were purchased from Sigma-Aldrich. Sodium butyrate $(\mathrm{NaB})$ was purchased from Millipore (Billerica, MA 01821, USA).

\section{Cell culture}

FTC133 FTC line; BCPAP, KTC-1, and TPC-1 PTC lines; and Hth7, Hth74, Hth83, Hth104, Hth112, and SW1736 ATC lines were studied. TAD-2 cells were kindly provided by Dr Michael Mingzhao Xing (The Johns Hopkins University School of Medicine). FTC133 cells were kindly provided by Dr Peter Goretzki (University of Leipzig, Germany) (Goretzki et al. 1990). BCPAP and KTC-1 cells were obtained from Drs Rebecca E Schweppe and Bryan Haugen (University of Colorado, Denver) with permission from the researchers who provided these lines to them. BCPAP cells were from Dr Massimo Santoro (Universita Federico II, Napoli, Italy), TPC-1 cells were kindly provided by Sissy Jhiang (The Ohio State University), and KTC-1 cells were from Dr Junichi Kurebayashi (Kawasaki Medical School, Japan) (Kurebayashi et al. 2000). The Hth7, Hth74, Hth83, Hth104, Hth112, and SW1736 cells were provided by Dr N-E Heldin (University Hospital, Uppsala, Sweden) (Dahlman et al. 2000). DNA profiling using the Identifier kit from Applied Biosystems (Carlsbad, CA, USA) confirmed that FTC133, BCPAP, and Hth83 cells were of thyroid origin and that HT29 cells (kindly provided by Dr M Ringel, The Ohio State University) were identical to the HT29 colon carcinoma cell line available from the ATCC. FTC133 and TAD-2 cells were grown in DMEM medium supplemented with $10 \%$ fetal bovine serum. The HT29, BCPAP, KTC-1, TPC-1, Hth7, Hth74, Hth83, Hth104, Hth112, and SW1736 cells were propagated in RPMI with $10 \%$ fetal bovine serum.

\section{NaB, TSA, and 5-Aza treatment}

Cells were plated in 24-well plates and grown for $24 \mathrm{~h}$ prior to treatment. 5-Aza was used at 5 and $10 \mu \mathrm{M}$ for $72 \mathrm{~h} . \mathrm{NaB}(5$ and $10 \mathrm{mM})$ and TSA $(1,2$, and $4 \mu \mathrm{M})$ were added for $24 \mathrm{~h}$ alone or following treatment with 5-Aza for $48 \mathrm{~h}$.

\section{Western blotting}

Protein extracts were prepared using non-reducing sample buffer $(62.5 \mathrm{mM}$ Tris $\mathrm{pH} 6.8,1 \mathrm{mM}$ sodium vanadate, $1 \mathrm{mM}$ sodium fluoride, $2 \%$ SDS, and $10 \%$ glycerol) (Millipore). Western blotting was performed as described previously (Tsygankova et al. 2004). Proteins were detected and analyzed using the FUJI LAS-3000 system and Multi Gauge 3.0 software (Fuji, Tokyo, Japan).

\section{siRNA transfection}

Scrambled siRNA (\#1027280) and RaplGAP siRNA duplexes 1 (\#SI01737043) and 2 (\#SI01737050) were purchased from Qiagen. Amaxa nucleofection was used to introduce siRNAs $(100 \mathrm{nM})$ into BCPAP and 
Hth83 cells. Following electroporation, cells were plated in 12 -well plates $\left(10^{5}\right.$ cells/well $)$ and $\mathrm{NaB}(5 \mathrm{mM})$, TSA $(1 \mu \mathrm{M})$, or DMSO (control) added for $24 \mathrm{~h}$.

\section{RT-PCR}

RNA was isolated using TRIzol (Invitrogen). RT-PCR was performed using the SuperScript III First-Strand Synthesis RT-PCR system (Invitrogen) according to the manufacturer's instructions. PCR products of Rap1GAP and $\beta$-actin were analyzed on $1.0 \%$ agarose gels and imaged using GelDoc XR and Quantity One 4.5.2 software (Bio-Rad).

\section{Rap2 activity}

Rap2 activity was assessed as described previously for Rap1 (Tsygankova et al. 2004).

\section{Statistical analyses}

All experiments were performed three or more times with similar results. Statistical analyses were performed using Excel 2007 software. Data are presented as means \pm s.D., and significance was assessed by $t$-test. A $P$ value $<0.05$ was considered to be statistically significant.

\section{Results}

\section{HDAC inhibitors increase Rap1GAP expression in thyroid cancer cells}

We screened ten thyroid cancer cell lines for the expression of Rap1GAP by western blotting. Rap1GAP expression was very low or undetectable in all cell lines (Fig. 1). Based on previous reports that Rap1GAP was silenced via an epigenetic mechanism

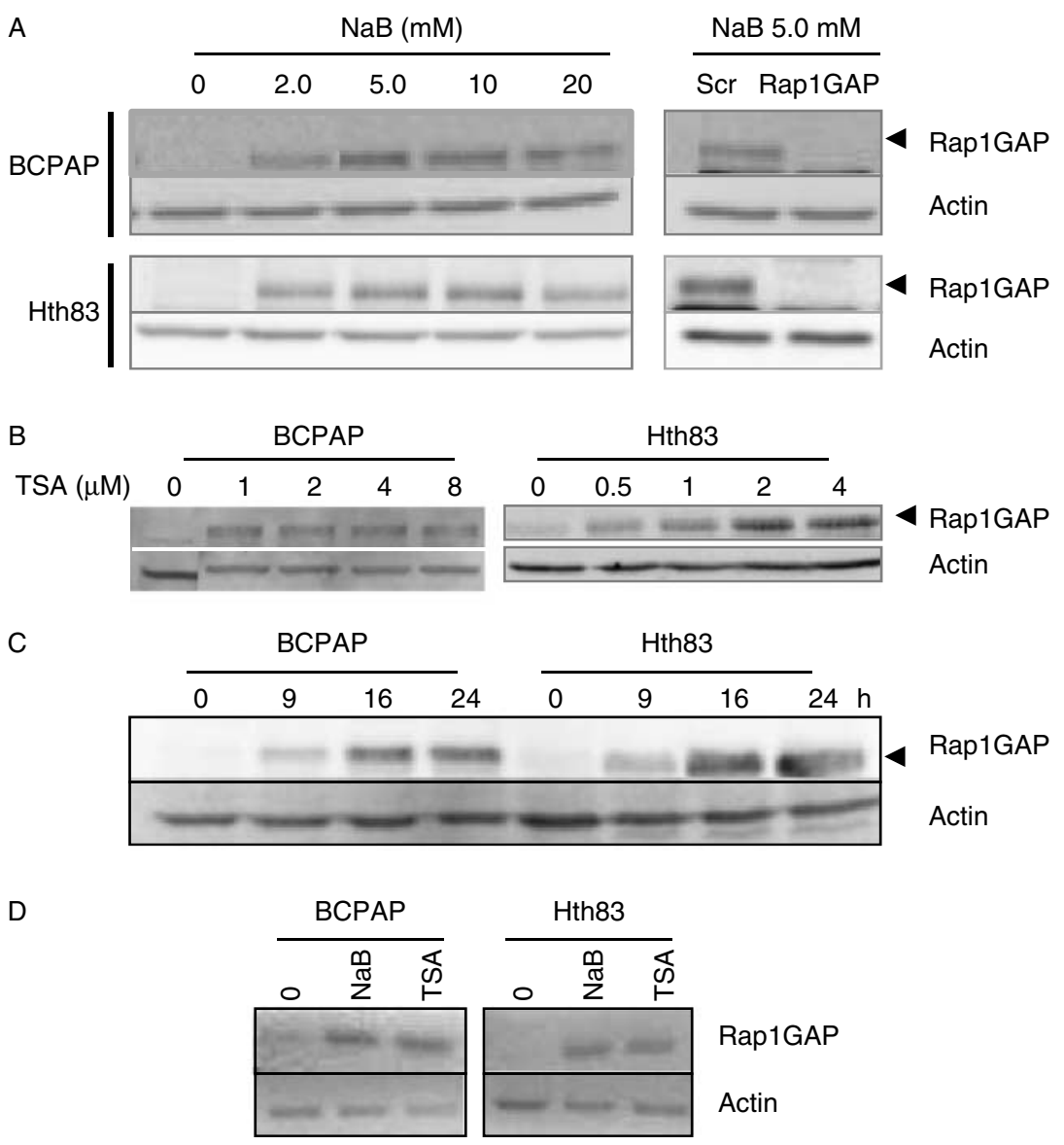

Figure 1 HDAC inhibitors upregulate Rap1GAP expression. (A) NaB (0, 2, 5, 10, and $20 \mathrm{mM}, 24 \mathrm{~h})$ dose-dependently induced Rap1GAP expression in BCPAP (PTC) and Hth83 (ATC) cells (left panel). Cells were disrupted and total cell proteins were analyzed by western blotting for Rap1GAP. The same filters were probed for actin to document equal protein loading. Silencing Rap1GAP by RNAi abolished the induced protein species, confirming that it was Rap1GAP (right panel). (B) TSA (at the concentrations indicated, $24 \mathrm{~h}$ ) induced Rap1GAP in BCPAP and Hth83 cells. (C) Time course for the induction of Rap1GAP expression by TSA (1.0 $\mu \mathrm{M})$ in BCPAP and Hth83 cells. (D) BCPAP and Hth83 cells were cultured in the presence or absence of NaB (5 mM) or TSA (1 $\mu \mathrm{M})$ for $18 \mathrm{~h}$. Total RNA was extracted from the cells and RT-PCR was performed. 
(Zheng et al. 2009, Zuo et al. 2010), we assessed whether the expression of endogenous Rap1GAP could be restored to thyroid tumor cell lines. Initial studies were carried out in one PTC cell line (BCPAP) and one ATC cell line (Hth83). In both the cell lines, treatment with the histone deacetylase inhibitor $\mathrm{NaB}$ increased Rap1GAP expression (Fig. 1A left panel). Rap1GAP expression was maximally induced with $5 \mathrm{mM} \mathrm{NaB}$. We confirmed that the induced species was Rap1GAP by transfecting cells with RaplGAP-directed siRNAs. As shown in Fig. 1A (right panel), transfection with Rap1GAP-directed but not scrambled siRNAs eliminated the induced protein species, confirming that it represents Rap1GAP.

To corroborate these findings, a structurally distinct HDAC inhibitor, TSA, was used. TSA $(0.5-8 \mu \mathrm{M})$ increased Rap1GAP expression in both the cell lines (Fig. 1B). As deacetylase inhibitors act via a transcriptional mechanism, we analyzed the time course over which TSA increased Rap1GAP expression. Rap1GAP expression was detected after $9 \mathrm{~h}$ of TSA treatment and increased at $16 \mathrm{~h}$ (Fig. 1C). This time course is consistent with a transcriptional effect. Indeed, $\mathrm{NaB}$ and TSA increased Rap1GAP message levels in both BCPAP and Hth83 cells (Fig. 1D). Histones play a central role in transcriptional regulation. Acetylation of lysine 14 in histone H3 is commonly seen in genes that are actively transcribed. As expected, $\mathrm{NaB}$ and TSA increased histone $\mathrm{H} 3$ acetylation at this site (Fig. 2). Collectively, these data indicate that treatment of PTC or ATC cells with deacetylase inhibitors is sufficient to increase the expression of Rap1GAP.

\section{Differential effects of HDAC inhibitors and demethylating agents in PTC and ATC cell lines}

Demethylating agents restored the expression of Rap1GAP in melanoma cell lines (Zheng et al. 2009). We analyzed whether the same was true in thyroid cancer cells. Treatment with the demethylating agent 5-Aza elicited only modest effects on Rap1GAP expression in BCPAP and Hth83 cells (Fig. 2). As expected, 5-Aza treatment had no effect on histone acetylation. Combined treatment of 5-Aza with HDAC inhibitors induced differential effects in the PTCversus ATC-derived cell lines. In BCPAP cells, HDAC inhibitors induced Rap1GAP expression to a similar extent in the absence or presence of the demethylating agent. In Hth83 cells, treatment with the demethylating agent enhanced the effects of HDAC inhibitors on Rap1GAP expression. This differs from a recent report that showed a profound induction of Rap1GAP by 5-Aza, which was decreased following combined treatment with TSA in Hth83 cells (Zuo et al. 2010). The reason for these differences is unclear but may be related to the source of the Hth83 cells. Our findings indicate that Rap1GAP expression is extinguished by multiple mechanisms that include alterations in histone acetylation and promoter methylation.

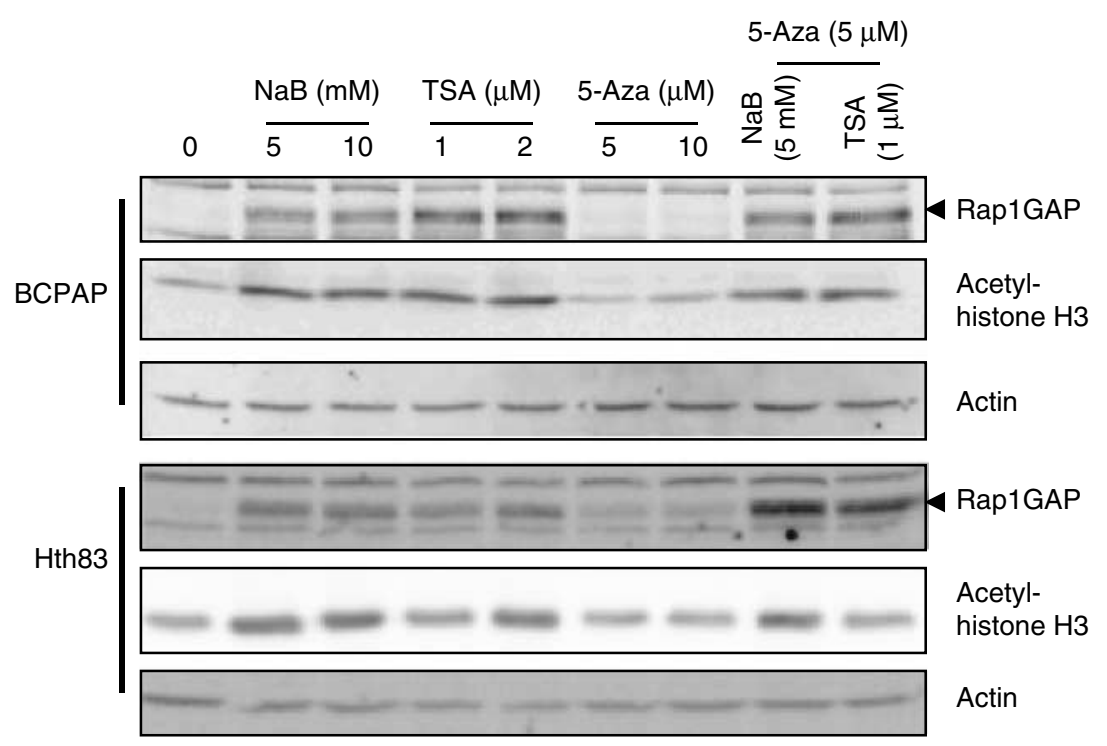

Figure 2 Differential effects of HDAC inhibitors and the demethylating agent 5-Aza in the induction of Rap1GAP. BCPAP and Hth83 cells were treated with NaB, TSA (24 h), 5-Aza (72 h), and HDAC inhibitors plus 5-Aza (72 h 5-Aza and $24 \mathrm{~h}$ HDAC inhibitors) as indicated. Protein was harvested for western blot analysis of Rap1GAP, acetyl-histone H3, and actin to confirm equal protein loading. 


\section{Widespread induction of Rap1GAP by HDAC inhibitors}

To further assess the utility of HDAC inhibitors in inducing the expression of Rap1GAP, we extended our analysis to eight additional thyroid cancer cell lines. Rap1GAP was poorly expressed in TPC-1 and KTC-1 cells and expressed at a slightly higher level in FTC133 cells (Fig. 3A). Treatment with HDAC inhibitors increased Rap1GAP expression in all the three cells lines. As seen in BCPAP cells, 5-Aza did not increase Rap1GAP expression in these lines. In addition, 5-Aza did not enhance the effects of the HDAC inhibitors on Rap1GAP expression.

Rap1GAP was undetectable in the Hth74, SWI736, Hth104, and Hth7 ATC cell lines. Low levels of Rap1GAP were detected in Hth112 cells. These cell lines could be subdivided into three groups, based on the effects of HDAC inhibitors and the demethylating

A

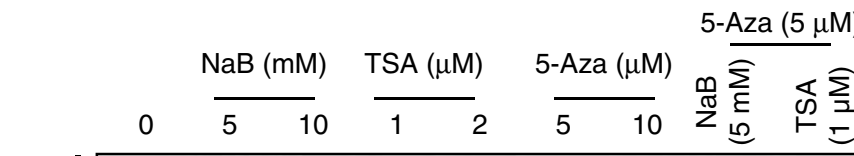
TPC-1

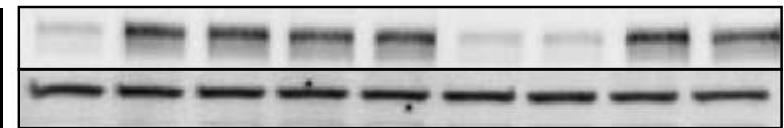
Rap1GAP Actin KTC-1

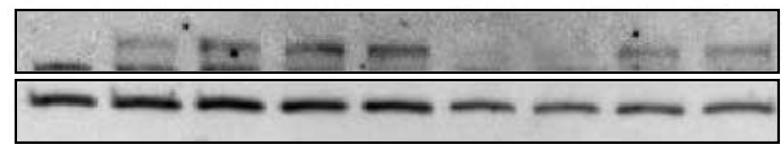

Rap1GAP

Actin

FTC133

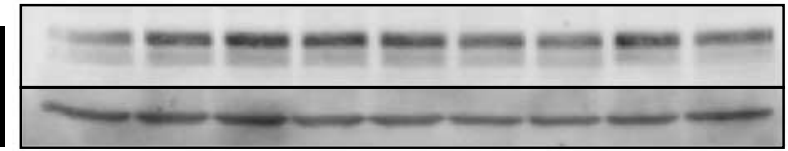

Rap1GAP

Actin

B

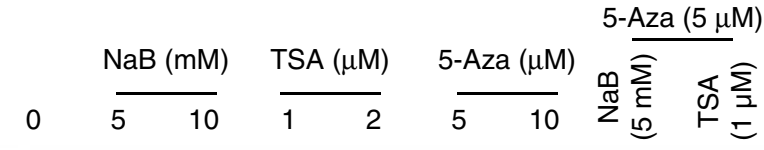
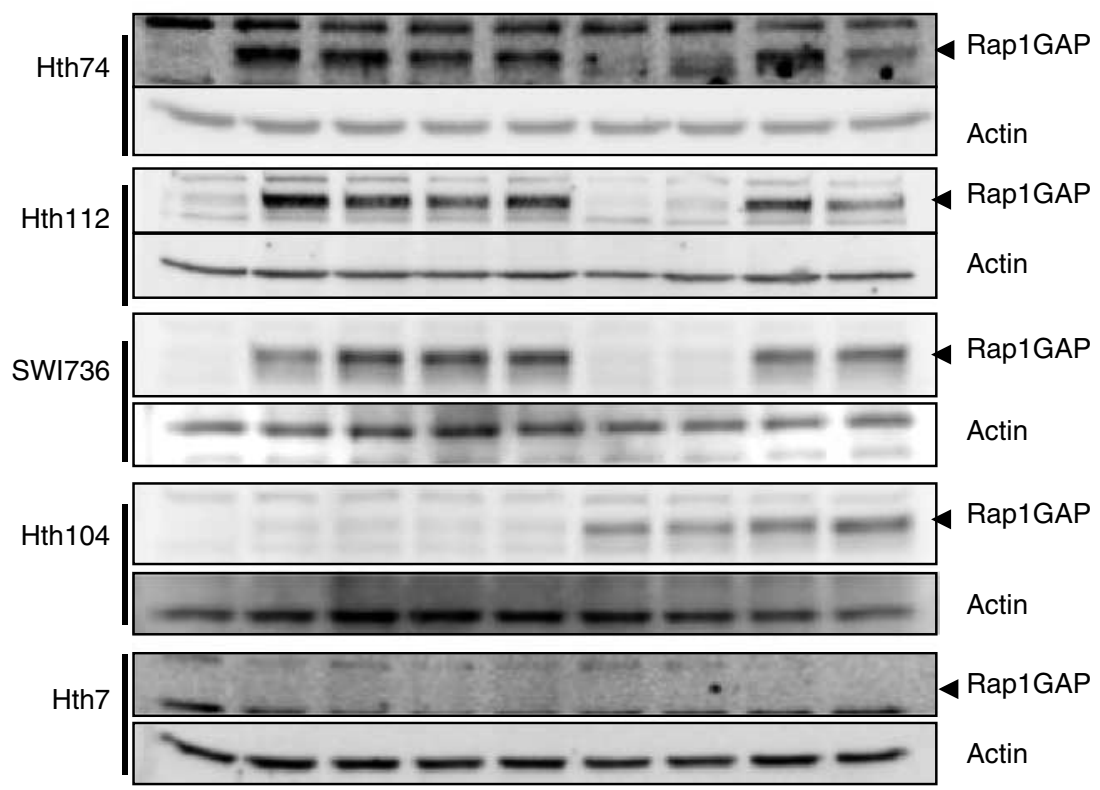

Figure 3 HDAC inhibitors increase Rap1GAP in a wide array of thyroid cancer cell lines. (A) Differentiated thyroid carcinoma cell lines TPC-1, KTC-1, and FTC133 were treated with NaB, TSA, and 5-Aza alone and in combination as indicated. Protein was harvested for the analysis of Rap1GAP and actin. (B) Anaplastic thyroid carcinoma cell lines Hth74, Hth112, SWI736, Hth104, and $\mathrm{Hth7}$ were treated with $\mathrm{NaB}, \mathrm{TSA}$, and 5-Aza alone and in combination as indicated. Protein was analyzed for Rap1GAP and actin expression. 
agent on Rap1GAP expression. In the first group, which included Hth74, Hth112, and SW1736 cells, NaB and TSA increased Rap1GAP expression, whereas 5-Aza had no effect (Fig. 3B). In addition, 5-Aza did not further increase Rap1GAP expression induced by HDAC inhibitors in these cells. In the second group, comprising the Hth104 cell line, 5-Aza but not $\mathrm{NaB}$ or TSA increased Rap1GAP expression. Inclusion of $\mathrm{NaB}$ or TSA modestly enhanced the effects of 5-Aza in this cell line. Hth7 cells reflected the third group. In this cell line, neither HDAC inhibitors nor demethylating agents restored Rap1GAP expression. It is possible that this cell line harbors a mutation in, or has deleted, the RaplGAP gene. Loss of heterozygosity for Rap1GAP has been reported in thyroid carcinomas (Nellore et al. 2008). In sum, these data indicate that epigenetic regulation of Rap1GAP is frequent in thyroid cancer cell lines and that HDAC inhibitors are more effective than demethylating agents in restoring Rap1GAP expression.

\section{HDAC inhibitors modulate Rap activity}

Downregulation of Rap1GAP in tumor cells is associated with sustained increases in Rap activity (Tsygankova et al. 2010). Increased Rap activity has been shown to enhance metastasis in breast and prostate cancer cell models (Itoh et al. 2007, Bailey et al. 2009). This implies that strategies to decrease Rap activity might harbor therapeutic potential. Hence, we explored if restoring endogenous Rap1GAP expression in tumor cells was sufficient to inactivate
Rap. Rap1 and Rap2 expression were analyzed by western blotting (Fig. 4A). Wistar rat thyroid (WRT) cells were included as a control for Rap1 (Tsygankova et al. 2001). Human colon carcinoma cells (HT29) were included as a control for Rap2 (Tsygankova et al. 2010). Rap2 expression was detected in BCPAP and Hth83 cells, and so we focused on the effects of HDAC inhibitors on Rap2 activity. BCPAP (Fig. 4B) and Hth83 (Fig. 4C) cells were treated with $\mathrm{NaB}$ and Rap2 activity analyzed by pull-down assay (Tsygankova et al. 2001). As expected, $\mathrm{NaB}$ increased the expression of Rap1GAP. Intriguingly, $\mathrm{NaB}$ significantly decreased Rap2 activity in both the cell lines (Fig. 4B and C). To corroborate these findings, the effects of TSA on Rap2 activity were examined. TSA also significantly decreased Rap2 activity (Fig. 4D and E). These data indicate that HDAC inhibitors may provide a tractable mechanism to inhibit Rap2 activity in human tumors.

To determine if the effects of HDAC inhibitors on Rap2 activity were dependent on the re-expression of Rap1GAP, we used siRNA to silence the expression of Rap1GAP. Transfection with RaplGAP-directed siRNAs significantly decreased, but did not abolish the induction of Rap1GAP by $\mathrm{NaB}$ in both the cell lines (Fig. 5A). Under these conditions, reducing Rap1GAP expression did not restore Rap2 activity in NaB-treated cells. Similar results were observed in cells stimulated with TSA (data not shown). To exclude the notion that HDAC inhibitors elicit non-specific effects on GTP binding or hydrolysis, we analyzed Rap2 activity in cells treated with $\mathrm{NaB}$ under conditions that did not
A

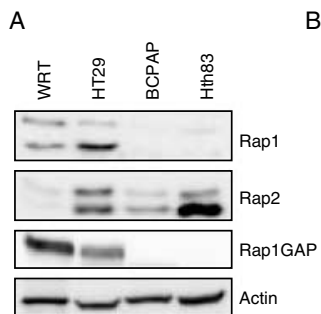

B

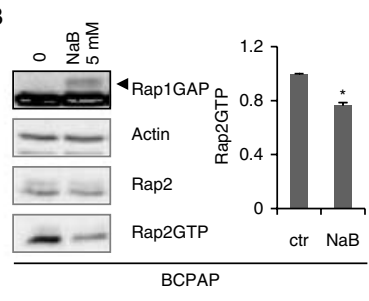

D

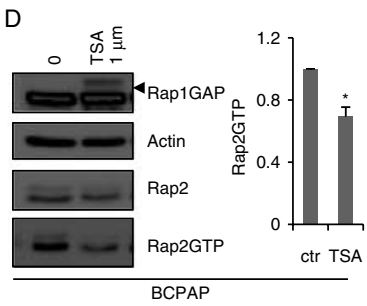

C

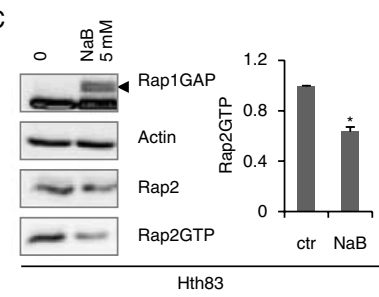

E

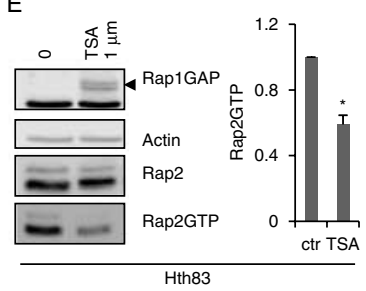

Figure 4 HDAC inhibitors decrease Rap2 activity. (A) Protein harvested from WRT, HT29, BCPAP, and Hth83 cells was analyzed by western blotting for Rap1, Rap2, Rap1GAP, and actin. (B and C) BCPAP and Hth83 cells were treated with NaB for $24 \mathrm{~h}$. Protein was analyzed for Rap1GAP, Rap2, and actin expression by western blotting and pull-down assay for Rap2 (Rap2GTP) activity. (D and E) BCPAP and Hth83 cells were treated with TSA for $24 \mathrm{~h}$ and analyzed as in panels $\mathrm{B}$ and $\mathrm{C}$. The results from three experiments are summarized $\left({ }^{\star} P<0.01\right)$. 
A
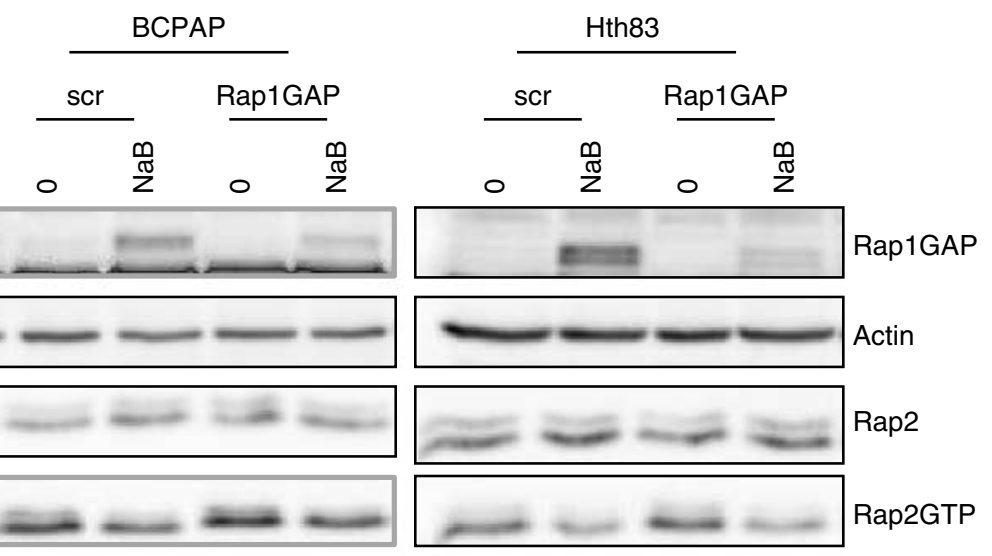

B
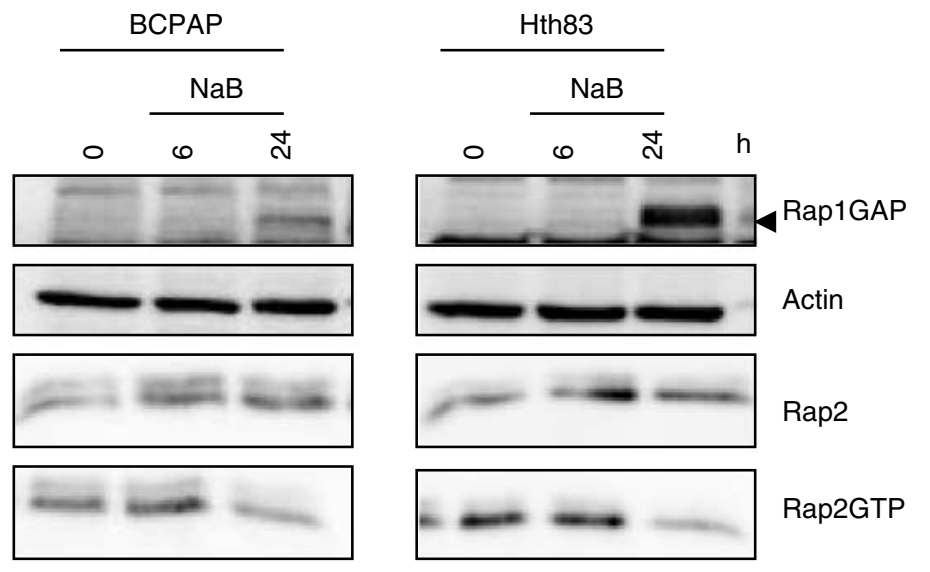

C

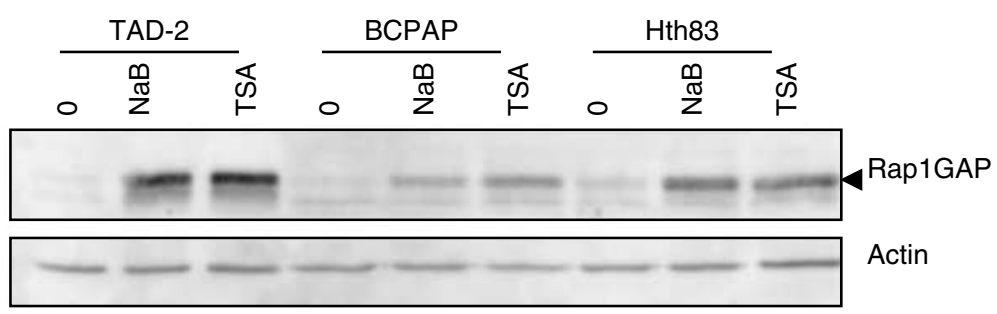

Figure 5 Silencing Rap1GAP expression has modest effects on Rap2 activity. (A) BCPAP and Hth83 cells were transfected with Rap1GAP-directed or scrambled (scr) siRNAs. At $24 \mathrm{~h}$ post-transfection, cells were treated with NaB (5 mM) for $24 \mathrm{~h}$. Cell lysates were subjected to pull-down assay for Rap2 activity (Rap2GTP) and total cell lysates were analyzed for Rap1GAP, Rap2, and actin expression. (B) BCPAP and Hth83 cells were treated with $\mathrm{NaB}(5 \mathrm{mM})$ for 6 and $24 \mathrm{~h}$. Rap2 activity (Rap2GTP) and Rap1GAP, Rap2, and actin expression were analyzed. (C) TAD-2, BCPAP, and Hth83 were treated with NaB (5 mM) or TSA (1 $\mu \mathrm{M})$ for $24 \mathrm{~h}$. Rap1GAP expression was analyzed by western blotting.

induce Rap1GAP expression. Rap1GAP expression was first detected after $9 \mathrm{~h}$ of treatment with the HDAC inhibitors (Fig. 1C). Thus, cells were stimulated with $\mathrm{NaB}$ for $6 \mathrm{~h}$ and Rap2 activity monitored. When added for $6 \mathrm{~h}$, neither $\mathrm{NaB}$ (Fig. 5B) nor TSA (data not shown) decreased Rap2 activity, although both agents decreased Rap2 activity at $24 \mathrm{~h}$ in the same experiments. These data suggest that the decrease in Rap2 activity is most likely due to alterations in gene expression, possibly including increased expression of Rap1GAP.

To assess whether the HDAC inhibitors restored Rap1GAP to levels approaching those seen in nontumorigenic thyroid cells, we compared Rap1GAP expression in BCPAP and $\mathrm{Hth} 83$ cells to that in immortalized human fetal thyroid cells (TAD-2 cells). Rap1GAP expression in thyroid tumor cell lines treated with HDAC inhibitors exceeded that seen in TAD-2 
cells (Fig. 5C). However, HDAC inhibitors increased Rap1GAP expression in these cells, suggesting that immortalization of these cells may have repressed the expression of Rap1GAP. Rap1GAP expression in WRT cells vastly exceeded than that in HDAC inhibitor-treated BCPAP and Hth83 cells (data not shown).

To further explore the mechanism underlying decreased Rap2 activity, we assessed whether HDAC inhibition altered the expression of other regulators of Rap2. SPA-1, a RapGAP that is enriched in hematopoietic cells, was not detected in BCPAP and Hth83 cells in the absence or presence of HDAC inhibition, although it was expressed in mouse spleen (Fig. 6A). Rap1GAPII, an alternatively spliced form of Rap1GAP that is expressed in WRT cells, was also not detected in the thyroid cancer cells lines. We were unable to detect the RapGEFs Epac1 or 2 in any of the cell lines (data not shown). However, the RapGEF C3G was expressed in BCPAP and Hth83 cells, where it was repressed by HDAC inhibition (Fig. 6B). These results indicate that HDAC inhibitors elicit effects on multiple Rap regulators that together culminate in decreased Rap2 activity.

A

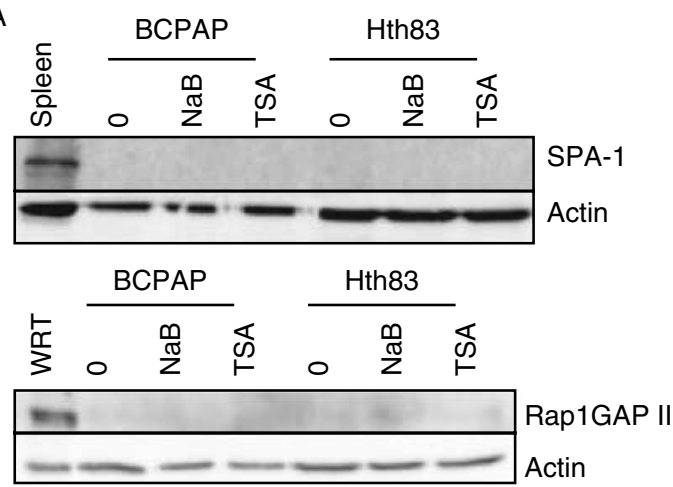

B

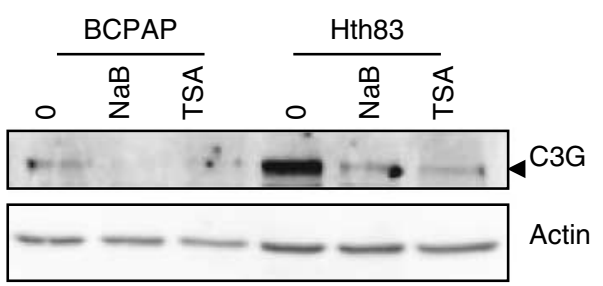

Figure 6 Effects of HDAC inhibition on Rap regulators. (A and B) BCPAP and Hth83 cells were stimulated with $\mathrm{NaB}(5 \mathrm{mM})$ and TSA $(1 \mu \mathrm{M})$ for $24 \mathrm{~h}$. Cells were disrupted and total cell proteins were analyzed by western blotting for (A) Spa-1, Rap1GAPII, and (B) C3G expression. The same filters were probed for actin to document equal protein loading.

\section{Discussion}

Rap1GAP is highly expressed in human thyroid follicular cells and downregulated in primary thyroid tumors (Tsygankova et al. 2007). Expression of Rap1GAP was further decreased in thyroid carcinomas versus adenomas, suggesting a role for Rap1GAP depletion in tumor progression (Nellore et al. 2008, Zuo et al. 2010). Silencing Rap1GAP expression in human colon cancer cell lines induced alterations in cell adhesion associated with the transition from adenoma to carcinoma (Tsygankova et al. 2010). Overexpression of Rap1GAP in B-cell lymphomas impaired the formation of metastatic liver nodules, but not the formation of primary tumors (Lin et al. 2010). Strikingly, the expression of Rap1GAP was markedly decreased in the small number of metastatic lesions that formed, whereas Rap1GAP expression was retained in primary tumors. Collectively, these studies show that downregulation of Rap1GAP in vitro induces alterations similar to those observed during tumor progression and that decreased expression of Rap1GAP may be required for metastasis. The goal of this study was to determine whether Rap1GAP expression could be restored in thyroid tumor cells.

HDACs 1 and 2 are overexpressed in thyroid carcinomas (Borbone et al. 2010). Based on this, we investigated whether HDAC inhibition restores the expression of Rap1GAP. The two structurally distinct HDAC inhibitors, NaB and TSA, were used. Both the inhibitors restored Rap1GAP expression in all PTC (three) and FTC (one) cell lines analyzed. HDAC inhibitors also induced Rap1GAP expression in four/six ATC cell lines. By contrast, treatment with the demethylating agent 5-Aza-deoxycytidine failed to increase Rap1GAP expression in PTC and FTC cells and restored Rap1GAP expression in a single ATC cell line. These results suggest that HDAC inhibition is a promising approach to restore Rap1GAP expression to thyroid tumor cells. These findings confirm those published recently, showing that TSA induced Rap1GAP expression in a PTC cell line (TPC-1; Zuo et al. 2010). In this report, treatment with 5-azacytidine but not TSA induced Rap1GAP in Hth83 cells. In our hands, 5-Aza had only modest effects on Rap1GAP expression in these cells, even when used at concentrations higher than those used by Zuo et al. The reasons for these differences are unclear. Nonetheless, both the studies indicate that Rap1GAP expression can be restored in thyroid tumor cells. Our results show that HDAC inhibitors provide the best strategy to restore Rap1GAP expression. 
Restoring Rap1GAP expression harbors therapeutic potential only if it is functionally significant. Importantly, treatment with $\mathrm{NaB}$ or TSA significantly decreased Rap2 activity in BCPAP and Hth83 cells. This suggests that HDAC inhibition provides a tractable approach to inhibit Rap activity in tumor cells. The mechanism underlying the decrease in Rap activity appears to be complex. Silencing Rap1GAP expression using RNA interference did not consistently restore Rap2 activity in cells treated with HDAC inhibitors. In BCPAP cells, silencing Rap1GAP increased Rap2 activity in one-third of the experiments performed. These findings may indicate that residual Rap1GAP expression in cells transfected with RaplGAP siRNAs is sufficient to inhibit Rap2 activity and that subtle differences in the efficiency of silencing explain the inconsistent effects on Rap2 activity. We did not observe any effects of silencing Rap1GAP on Rap2 activity in Hth83 cells where we also failed to abolish Rap1GAP expression. We did not detect the expression of other RapGAPs, including SPA-1 and Rap1GAPII, in the thyroid cancer cell lines either in the absence or in the presence of HDAC inhibitors. Therefore, it seems unlikely that the induction of other RapGAPs is responsible for decreased Rap2 activity. Rap activity reflects the balance in the expression and activities of RapGEFs and RapGAPs. We examined the expression of RapGEFs. We were unable to detect Epac1 or 2 in these cells, which may relate to the quality of the antibodies used. Interestingly, the expression of $\mathrm{C} 3 \mathrm{G}$ was decreased by HDAC inhibition. In principle, this provides a second mechanism through which HDAC inhibitors decrease Rap activity. Rap1 activity promotes metastasis in human breast and prostate cancer cells (Itoh et al. 2007, Bailey et al. 2009). Rap activity is required for RET/PTC1-induced BRAF activation, mitogenesis and cytoskeletal reorganization in thyroid cells (De Falco et al. 2007). Hence, strategies to inhibit Rap activity may be of therapeutic utility in a wide array of human tumors.

\section{Declaration of interest}

The authors declare that there is no conflict of interest that could be perceived as prejudicing the impartiality of the research reported.

\section{Funding}

This work was supported by public health service grant CA127986 awarded to J L Meinkoth.

\section{Acknowledgements}

We thank Oxana M Tsygankova and Lisa A Vuchak for helpful suggestions with experimental design.

\section{References}

Bailey CL, Kelly P \& Casey PJ 2009 Activation of Rap1 promotes prostate cancer metastasis. Cancer Research 69 4962-4968. (doi:10.1158/0008-5472.CAN-08-4269)

Borbone E, Berlingieri MT, De Bellis F, Nebbioso A, Chiappetta G, Mai A, Altucci L \& Fusco A 2010 Histone deacetylase inhibitors induce thyroid cancerspecific apoptosis through proteasome-dependent inhibition of TRAIL degradation. Oncogene 29 105-116. (doi:10.1038/onc.2009.306)

Dahlman T, Lammerts E, Wik M, Bergström D, Grimelius L, Westermark K, Rubin K \& Heldin NE 2000 Fibrosis in undifferentiated (anaplastic) thyroid carcinomas: evidence for a dual action of tumour cells in collagen type I synthesis. Journal of Pathology 191 376-386. (doi:10.1002/1096-9896(2000)9999:9999<::AIDPATH643 > 3.0.CO;2-W)

De Falco V, Castellone MD, De Vita G, Cirafici AM, Hershman JM, Guerrero C, Fusco A, Melillo RM \& Santoro M 2007 RET/papillary thyroid carcinoma oncogenic signaling through the Rap1 small GTPase. Cancer Research 67 381-390. (doi:10.1158/0008-5472. CAN-06-0981)

Gao Q, Srinivasan S, Boyer SN, Wazer DE \& Band V 1999 The E6 oncoproteins of high-risk papillomaviruses bind to a novel putative GAP protein, E6TP1, and target it for degradation. Molecular and Cellular Biology 19 733-744.

Gao Q, Singh L, Kumar A, Srinivasan S, Wazer DE \& Band V 2001 Human papillomavirus type 16 E6-induced degradation of E6TP1 correlates with its ability to immortalize human mammary epithelial cells. Journal of Virology 75 4459-4466. (doi:10.1128/JVI.75. 9.4459-4466.2001)

Gao X, Zhang Y, Arrazola P, Hino O, Kobayashi T, Yeung RS, Ru B \& Pan D 2002 Tsc tumour suppressor proteins antagonize amino-acid-TOR signalling. Nature Cell Biology 4 699-704. (doi:10.1038/ncb847)

Goretzki PE, Frilling A, Simon D \& Roeher HD 1990 Growth regulation of normal thyroids and thyroid tumors in man. Recent Results in Cancer Research 118 48-63.

Ishida D, Kometani K, Yang H, Kakugawa K, Masuda K, Iwai K, Suzuki M, Itohara S, Nakahata T, Hiai H et al. 2003 Myeloproliferative stem cell disorders by deregulated Rap1 activation in SPA-1-deficient mice. Cancer Cell 4 55-65. (doi:10.1016/S1535-6108(03)00163-6)

Itoh M, Nelson CM, Myers CA \& Bissell MJ 2007 Rap1 integrates tissue polarity, lumen formation, and tumorigenic potential in human breast epithelial cells. Cancer Research 67 4759-4766. (doi:10.1158/0008-5472.CAN06-4246)

Kurebayashi J, Tanaka K, Otsuki T, Moriya T, Kunisue H, Uno M \& Sonoo H 2000 All-trans-retinoic acid modulates 
expression levels of thyroglobulin and cytokines in a new human poorly differentiated papillary thyroid carcinoma cell line, KTC-1. Journal of Clinical Endocrinology and Metabolism 85 2889-2896. (doi:10.1210/jc.85.8.2889)

Lin KB, Tan P, Freeman SA, Lam M, McNagny KM \& Gold MR 2010 The Rap GTPases regulate the migration, invasiveness and in vivo dissemination of B-cell lymphomas. Oncogene 29 608-615. (doi:10.1038/onc. 2009.345)

McLeod SJ, Shum AJ, Lee RL, Takei F \& Gold MR 2004 The Rap GTPases regulate integrin-mediated adhesion, cell spreading, actin polymerization, and Pyk2 tyrosine phosphorylation in B lymphocytes. Journal of Biological Chemistry 279 12009-12019. (doi:10.1074/jbc. M313098200)

Nagai H, Negrini M, Carter SL, Gillum DR, Rosenberg AL, Schwartz GF \& Croce CM 1995 Detection and cloning of a common region of loss of heterozygosity at chromosome 1p in breast cancer. Cancer Research $\mathbf{5 5}$ 1752-1757.

Nellore A, Paziana K, Ma C, Tsygankova OM, Wang Y, Puttaswamy K, Iqbal AU, Franks SR, Lv Y, Troxel AB et al. 2008 Loss of Rap1GAP in papillary thyroid cancer. Journal of Clinical Endocrinology and Metabolism 94 1026-1032. (doi:10.1210/jc.2008-1042)

Tsygankova OM, Saavedra A, Rebhun JF, Quilliam LA \& Meinkoth JL 2001 Coordinated regulation of Rap1 and thyroid differentiation by cyclic AMP and protein kinase A. Molecular and Cellular Biology 21 1921-1929. (doi:10.1128/MCB.21.6.1921-1929.2001)

Tsygankova OM, Feshchenko E, Klein PS \& Meinkoth JL 2004 Thyroid-stimulating hormone/cAMP and glycogen synthase kinase 3 \{beta\} elicit opposing effects on Rap1GAP stability. Journal of Biological Chemistry 279 5501-5507. (doi:10.1074/jbc.M305824200)

Tsygankova OM, Prendergast GV, Puttaswamy K, Wang Y, Feldman MD, Wang H, Brose MS \& Meinkoth JL 2007 Downregulation of Rap1GAP contributes to Ras transformation. Molecular and Cellular Biology 27 6647-6658. (doi:10.1128/MCB.00155-07)

Tsygankova OM, Ma C, Tang W, Korch C, Feldman MD, Lv Y, Brose MS \& Meinkoth JL 2010 Downregulation of
Rap1GAP in human tumor cells alters cell/matrix and cell/cell adhesion. Molecular and Cellular Biology 30 3262-3274. (doi:10.1128/MCB.01345-09)

Tuttle RM, Leboeuf R \& Martorella AJ 2007 Papillary thyroid cancer: monitoring and therapy. Endocrinology and Metabolism Clinics of North America 36 753-778 vii. (doi:10.1016/j.ecl.2007.04.004)

Vuchak LA, Tsygankova OM, Prendergast GV \& Meinkoth JL 2009 Protein kinase A and B-Raf mediate extracellular signal-regulated kinase activation by thyrotropin. Molecular Pharmacology 76 1123-1129. (doi:10.1124/ mol.109.060129)

Williamson C, Pannett AA, Pang JT, Wooding C, McCarthy M, Sheppard MN, Monson J, Clayton RN \& Thakker RV 1997 Localisation of a gene causing endocrine neoplasia to a $4 \mathrm{cM}$ region on chromosome 1p35-p36. Journal of Medical Genetics 34 617-619. (doi:10.1136/jmg.34.8.617)

Zhang L, Chenwei L, Mahmood R, van Golen K, Greenson J, Li G, D’Silva NJ, Li X, Burant CF, Logsdon CD et al. 2006 Identification of a putative tumor suppressor gene Rap1GAP in pancreatic cancer. Cancer Research 66 898-906. (doi:10.1158/0008-5472.CAN-05-3025)

Zheng H, Gao L, Feng Y, Yuan L, Zhao H \& Cornelius LA 2009 Down-regulation of Rap1GAP via promoter hypermethylation promotes melanoma cell proliferation, survival, and migration. Cancer Research 69 449-457. (doi:10.1158/0008-5472.CAN-08-2399)

Zuo H, Gandhi M, Edreira MM, Hochbaum D, Nimgaonkar VL, Zhang P, DiPaola J, Evdokimova V, Altschuler DL \& Nikiforov YE 2010 Downregulation of Rap1GAP through epigenetic silencing and loss of heterozygosity promotes invasion and progression of thyroid tumors. Cancer Research 70 1389-1397. (doi:10.1158/0008-5472. CAN-09-2812)

Received in final form 18 February 2011 Accepted 2 March 2011 Made available online as an Accepted Preprint 2 March 2011 